\title{
Non-Traditional Approaches to Diagnosis and Management of Type-2 Diabetes Mellitus: Point of View
}

\section{Gundu HR Rao*}

Emeritus Professor, Laboratory Medicine and Pathology, University of Minnesota, USA

Modern medicine has failed to reduce or prevent the rapid increase in Cardio-Metabolic Disorders (CMDs) worldwide. According experts at NIH/USA, the disease driven approach to healthcare, has resulted in spiraling costs, as well as a fragmented health system. Current Diagnostic methodologies identify "at risk" patients at a point that is too late, to account for the damage already caused by underlying pathologies. For instance, if we consider the type- 2 diabetes (T2D) detection, monitoring standards such as HbAlc, fasting blood glucose, aid in identifying altered levels of glucose in circulating blood. However, using these markers would result in providing just palliative treatment of the disease and its symptoms, whereas, the damage already done is not reversible. We strongly feel that early detection of the underlying pathologies, and effective management of the observed risks or dysfunctions, is the best choice for reducing the health care costs. Non-invasive methods are available, to monitor blood glucose, autonomic and peripheral sympathetic neuropathy, retinopathy, endothelial dysfunction and 3D imaging of carotid artery. Using one such platform (TM-Oxi), over 10,000 subjects, have been screened in various Lifespan clinics in India. Use of such technology seems to have identified autonomic, sudo-motor and endothelial dysfunction reliably, to distinguish diabetic populations from non-diabetic subjects. However, use of such new technologies, have not been tested, validated and adapted widely. Non-traditional approaches to the management of health care, which is called the integrative approach, emphasizes prevention, health maintenance and early intervention, and utilizes all appropriate evidence-based and personalized therapeutic approaches, to achieve optimal health and wellbeing across one's lifespan. In this overview or point of view, I present some novel approaches for the diagnosis of the risks as well as management of Clinical complications associated withType- 2 diabetes.

Type-diabetes is a serious public health problem of Global Importance and poses great economic burden to all the Nations. However, Developing Nations and resource poor countries bear much greater economic burden in view of greater prevalence of this chronic metabolic disease. According the International Diabetes Federation, 387 million people have diabetes worldwide; by 2035 this number will rise to 592 million. The number of people with type-2 diabetes is increasing in every country $[1,2]$. In the Global Diabetic population, greater than $75 \%$ of Type- 2 diabetes subjects live in resource poor countries. The greatest numbers of patients with diabetes are between 40 and 59 years of age. Close to 200 million people with diabetes are undiagnosed. Diabetes caused 4.9 million deaths in 2014; every seven seconds a person dies from diabetes related complication. Diabetes caused at least USD 612 billion dollars in health expenditure in 2014 ( $11 \%$ of total spending on adults). With over 65 million diabetics and an equal number of pre-diabetics, India, is considered the diabetes capital of the world and China is closely approaching this number to claim the title. Vascular complications related to diabetes accounts for the majority of economic burden worldwide. About one half of the diabetics die prematurely due to cardiovascular complications and a tenth due to renal failure. In spite of the fact that there is an increase in the incidence of this disease worldwide, the major share of the morbidity and mortality is predicted to occur in the resource poor countries. Various risk factors that promote diabetes and vascular disease are known. Although these risks are modifiable or preventable, there are very little efforts in progress in majority of the developing world, to monitor or control them.

By and large the gold standard diagnostic method used for the detection of diabetes is measurement of fasting blood glucose or impaired glucose metabolism or glycated hemoglobin (HbA1c). In developing countries as well as resource poor countries there is no an organized surveillance program or effective management programs for these cardio-metabolic disorders. For instance in a country like India, which is considered the diabetes capital of the world, there is no national platform to address these chronic diseases. In addition, in the absence of health insurance, people do not go for regular annual checkups. Therefore, by the time they discover that they have diabetes or impaired glucose tolerance/insulin resistance, they will have already had considerable irreversible vascular damage.

According to some studies, early onset diabetes is more aggressive than the late onset diabetes. If so, we need to consider as to how early we should start the prevention of this chronic disease. In India, for instance, even to this day $30 \%$ of the children born are of low birth weight. Pioneering studies done at the Mission Hospital Mysore, India, on "Mysore Cohort" as well as at Pune, India, by Yajnik and associates have clearly demonstrated that these low birth weight children are predisposed for the development of metabolic disorders [3-6]. Therefore, we need to start management and prevention of this disease with better maternal nutrition. We also need to address the nutrition of the neonates as well as the adolescents. In addition, we need to find out the mechanisms by which excess blood glucose and insulin levels modulate the patho-physiology and function of blood vessels.

Not only diabetes care is in disarray, but also our understanding of the mechanisms underlying clinical complications associated with this disease is in disarray. By and large, the major goals in caring for patients with diabetes are to prevent, or at least slow, the development of clinical complications such as; Micro-vascular (eye and kidney disease) accomplished through control of blood sugar and blood pressure; Macro-vascular (coronary, cerebrovascular,

*Corresponding author: Gundu HR Rao, Emeritus Professor, Laboratory Medicine and Pathology, University of Minnesota Hospital, Clinic Box 609, University of Minnesota, MN-55455, USA, Tel: 952594 5248; E-mail: gundurao9@gmail.com

Received December 03, 2014; Accepted December 31, 2014; Published January 05, 2015

Citation: Rao GHR (2015) Non-Traditional Approaches to Diagnosis and Management of Type-2 Diabetes Mellitus: Point of View. J Diabetes Metab 6: 489 doi:10.4172/2155-6156.1000489

Copyright: (c) 2015 Rao GHR. This is an open-access article distributed under the terms of the Creative Commons Attribution License, which permits unrestricted use, distribution, and reproduction in any medium, provided the original author and source are credited. 
Citation: Rao GHR (2015) Non-Traditional Approaches to Diagnosis and Management of Type-2 Diabetes Mellitus: Point of View. J Diabetes Metab 6: 489. doi:10.4172/2155-6156.1000489

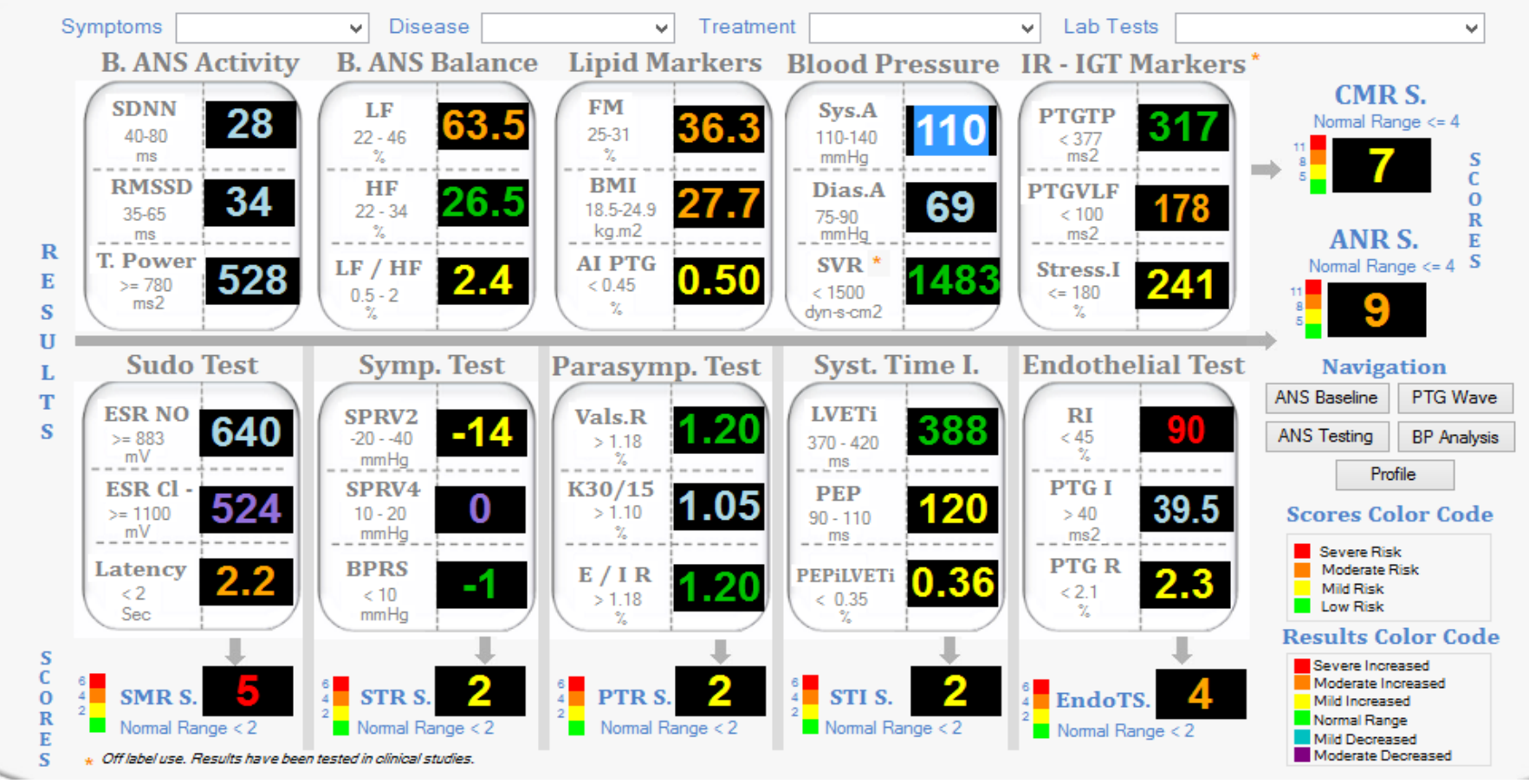

PATIENT PROFILE: RISK FACTORS CHART AND FOLLOW UP

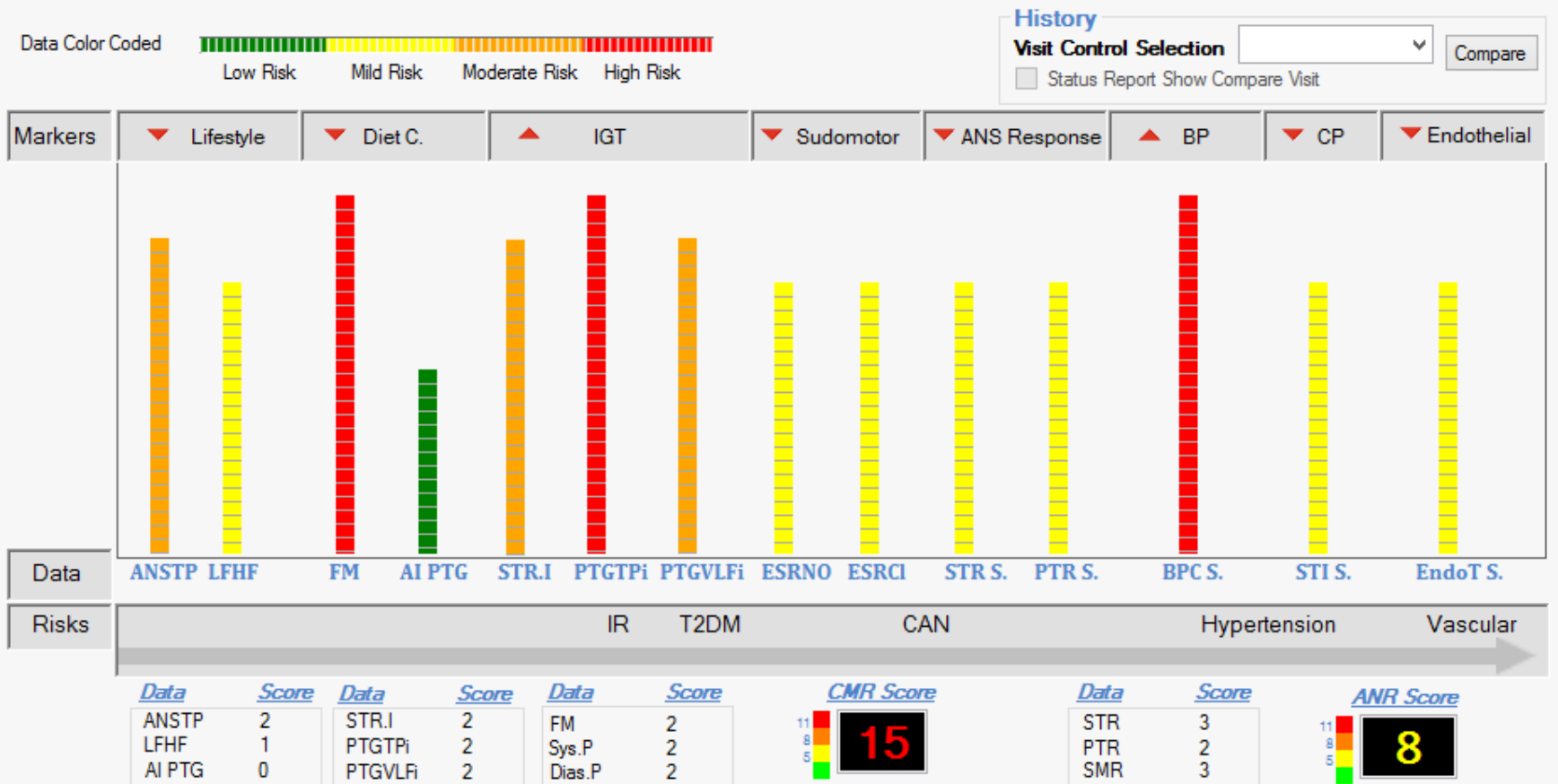

Figure 1: The scores for various functional tests.

peripheral vascular) through control of lipids, hypertension and smoking. However, we do not know how the elevated levels of blood glucose, circulating insulin and altered blood pressure modulate the pathophysiology of the blood vessels and induces severe dysfunction of this organ system. For instance obesity has been associated with type-2 diabetes for several decades. The major basis for this link is the ability of obesity related altered metabolism with insulin resistance. Insulin resistance is linked with a variety of disorders such as hypertension, hyperlipidemia, atherosclerosis, and metabolic syndrome. According to some recent studies obesity may be a proxy than the cause for insulin resistance. In the USA alone, there are 30 million individuals, who are obese with no insulin resistance. On the other hand, there are 6 million lean people who have insulin resistance. Little is know about how the foods we eat modulate the gene expression in terms of proteomics and 
metabolomics. These are very important problems. However, scientific studies can provide answers to such complicated questions.

There is a great need for the development of a non-invasive comprehensive diagnostic platform, for early detection of cardiometabolic disorders such as, hypertension, central abdominal obesity, metabolic syndrome, Type-2 diabetes, various vasculopathies, heart disease and stroke. Several studies have demonstrated the usefulness of monitoring health of the blood vessels by using techniques that measure, Intima-Media Thickness (IMT), Pulse Wave Velocity (PWV), Ankle-Brachial Index (ABI), Carotid Duplex Ultrasound (CDU) Index, and Vessel Wall Plaque Volume (3D Carotid Ultrasound). Although many methodologies are available for early detection of altered vascular function, the most widely used method is monitoring aortic pulse wave (PWV). With the advance in technologies, now it is possible to use multiple technologies and build a comprehensive risk assessment platform. It is also possible to add capabilities for risk prediction in such a platform. Availability of such a diagnostic platform will facilitate the surveillance of cardio-metabolic disorders at the community level.

In the meantime, we are looking at the available POC devices worldwide, which can be used for early detection of cardio-metabolic disorders such as hypertension, glucose intolerance, type-2 diabetes and its clinical complications. LD Technologies of Miami, Florida (www.ldteck.com) specializes in the development of non-invasive Point-Of-Care devices. They have put together a platform called "R.I.S.C", (for Life Span, India), which uses Autonomic Nervous System (ANS) tests, Heart Rate Variation (HRV) tests, SudoMotor Function tests (TM-Oxi system). The diagnostic platform used at the IPC Heart Care (TM-Oxi), Mumbai and the Life Span Kiosks (R.I.S.C) in different cities in India, is a combination of many devices and uses photoplethysmography, spectrophotometry, oscillometry and galvanic skin response technologies and displays data rapidly. Since these timetested devices have been used to obtain information on the pulse wave velocity of macro and micro vessels, with appropriate proprietary algorithms one can obtain information on the health of these vessels. Based on such information, the scores for various functional tests are obtained, digitized and displayed color-coded (green orange, yellow and red), and printed out graphically as well as digitally. It generates impressive diagnostic reports with some recommendations (Figure 1).

Lifespan chain of clinics in India, which uses this platform (TMOxi) under the brand-name R.I.S.C (Report on Insulin Sensitivity and Control) recently summarized the results of their study on over 10,000 subjects and claimed that $80 \%$ of diabetic Indians are at high risk of heart disease. Their advertisement in the Times of India a leading News Paper claims, that this platform is capable of providing a complete assessment of over 30 cardio metabolic indicators. What we need today is a combination of such POC methodologies, for early detection of the altered physiology and function of blood vessels. Since this is a platform that uses non-invasive methodologies, we could add to this platform additional features such as, ability to detect blood glucose, ability to monitor the plaque volume in carotid arteries by $3 \mathrm{D}$ ultrasound, ability to monitor the vascular physiopathology of vessels of the eye and follow morphology of the endothelial surface of these vessels, for any plaque build up by Optical Coherence Tomography (OCT). Based on such capabilities, develop risk profiling and risk prediction algorithms for following the progress of the vessel wall diseases. One can also include a fundus camera with appropriate software embedded programs to assess diabetic retinopathy. In addition to these POC devices, we should also develop the ability to integrate other observed risk factors to the data collected from such devices, for improving the risk score, so that one could use such comprehensive approach, to the early detection of vascular dysfunction, follow the progression and regression of the vascular disease and manage these diseases effectively.

Earlier clinical trials have demonstrated that the tight control of hyperglycemia reduces or prevents micro-vascular complications such as retinopathy, nephropathy and neuropathy. However, these studies have not demonstrated significant benefits on macro-vascular lesions leading to acute vascular events such as myocardial infarction and stroke. Therefore, it is worthwhile to develop novel management strategies to prevent or reduce macro-vascular complications. In terms of novel management strategies, we at the IPC Heart Care, Mumbai, India (www.ipcheartcenter.com) are evaluating, green tea of mulberry as well as green tea from regular tea leaves as supplements to mange postprandial hyperglycemia. A major green tea polyphenol, epigallocatechin-3-gallate, inhibits obesity, metabolic syndrome and fatty liver in high-fat-fed mice model. In addition to the tea mentioned above, we also are evaluating D-ribose, L-arginine, Curcumin, Carnitine, Resveratrol, Anthocyanin-rich blue-berry extract, citrusderived flavonoid Narangenin, grape seed extract Procyanidon, green tea extract Tegreen, mulberry leaf extract 1-deoxynojirimcin (DNJ), water soluble extract of cinnamon and coffee (Green Coffee), and hibiscus tea. Based on the results of our studies, we plan to develop a "poly-pill" with indigenous phyto-chemicals for the management of postprandial hyperglycemia. Since we use TM-Oxi system (LDTechnologies, Miami Florida) to monitor risk profile of patients, we can evaluate various indigenous therapies for their effectiveness or otherwise, in managing the observed risks of metabolic syndrome, $\mathrm{T}-2 \mathrm{D}$ and cardiovascular diseases, using this non-invasive diagnostic platform.

\section{References}

1. Mohan V, Rao GH (2007) Type-2 Diabetes in South Asians: Epidemiology, Risk Factors and Prevention. Jaypee Medical Publishers, New Delhi, India.

2. Gandhi PG, Rao GH (2014) The Spectral Analysis of Plethysmography to evaluate independent cardiovascular risk factor. International Journal of General Medicine (In Press).

3. Yajnik CS (2007) The lifecycle effects of nutrition and body size on adult adiposity, diabetes and cardiovascular disease. Obes Rev 3: 217-224.

4. Yajnik CS (2003) Neonatal anthropometry: the thin-fat Indian baby. The Pune nutritional study. Int J Obes Related Metabolic Disorders 27: 173-180.

5. Yajnik CS (2004) Early life origins of insulin resistance and type-2 diabetes in India and other countries. J Nutr 134: 205-210.

6. Yajnik CS (2004) Obesity epidemic in India: intrauterine origins? Proc. Nutr. Soc 63: 387-396. 